\title{
Comparison of Complex Moduli Obtained from Forced and Free Damped Oscillations
}

\author{
R. A. HELLER, Institute for the Study of Fatigue and \\ Reliability, Columbia University, New York, New York 10027, \\ and C. J. NEDERVEEN, T.N.O., Delft, Netherlands
}

\begin{abstract}
Synopsis
While the concept of the complex modulus is based on a forced vibration experiment it is a frequent practice to perform instead a much simpler free damped oscillation test from which an approximate value of the modulus is then evaluated. The validity of this approach and the ensuing errors are discussed and illustrated on a multielement generalized Maxwell body. It is shown that with modifications the method is applicable even for highly damped materials.
\end{abstract}

\section{Introduction}

The definition of the complex modulus is based on a forced oscillation experiment in which the lag angle between the imposed sinusoidal strain and the resulting sinusoidal stress as well as the shape of the stress-strain diagram are measured. ${ }^{1}$ Because the measurement of a lag angle is rather difficult it is a frequent practice to perform, instead, a free damped vibration experiment measuring the natural frequency and the logarithmic decrement from which an approximate alue of the complex modulus is then computed. ${ }^{2}$

It is to be noted, however, that the decaying oscillations are no longer sinusoidal and, consequently, results obtained from them are inexact, the approximations being less and less accurate with increasing damping. ${ }^{3,4}$

The difference between results obtained in the two types of experiment will be analyzed with the assumption that a generalized Maxwell body, having a large number of spring and dashpot elements, can adequately represent the behavior of a real material. The method has 
been utilized for simple models before $;^{5,6}$ a more realistic example will be discussed here.

The complex modulus

$$
G^{*}=G(1+j \tan \delta)
$$

is the constant of proportionality between an imposed sinusoidal stress

$$
\sigma=\sigma_{0} \exp j \omega t
$$

and the resulting deformation

$$
\gamma=\frac{\sigma}{G^{*}}=\frac{\sigma_{0}}{\left|G^{*}\right|} \exp j(\omega t-\delta)
$$

In the free vibration case a mass $m$ is attached to the model. For very soft materials an external spring with spring constant $S$ is also added to the model and one of two second-order differential equations

$$
m \ddot{\gamma}+\left[G^{-}\left(1+j \tan \delta^{-}\right)+S\right] \gamma=0
$$

or

$$
m \ddot{\gamma}+\left[G^{+}\left(1+j \tan \delta^{+}-(\lambda / 2) \tan \delta^{+}+S\right] \gamma=0\right.
$$

is solved with suitable initial conditions where $G^{-}, G^{+}$, and $\tan \delta^{-}$ or $\tan \delta^{+}$of eqs. (1.4) and (1.5) are not necessarily identical with $G^{*}$ and $\tan \delta$ of eq. (1.1).

The solution of eqs. (1.4) and (1.5)

$$
\gamma=C \exp j \omega t(1+j \lambda / 2)
$$

when substituted into eq. (1.4) yields ${ }^{7}$

$$
G^{-}=m \omega^{2}\left(1-\lambda^{2} / 4\right)-S
$$

and

$$
\tan \delta^{-}=\frac{\lambda}{1-\lambda^{2} / 4-S / m \omega^{2}}
$$

where $\lambda$ may be obtained from the measured logarithmic decrement, $\Delta$ as

$$
\Delta=\pi \lambda
$$


Several authors ${ }^{3.8}$ have debated the validity of eqs. (1.4), (1.7), and (1.8) and use, instead, eq. (1.5) which yields

$$
G^{+}=m \omega^{2}\left(1+\lambda^{2} / 4\right)-S
$$

and

$$
\tan \delta^{+}=\frac{\lambda}{1+\lambda^{2} / 4-S / m \omega^{2}}
$$

when damping is small the square term in eqs. (1.7), (1.8), (1.10), and (1.11) is neglected. Denoting the corresponding modulus and damping by $G^{\prime}$ and $\tan \delta^{\prime}$, respectively, the following equations result

$$
G^{\prime}=m \omega^{2}-S
$$

and

$$
\tan \delta^{\prime}=\frac{\lambda}{1-S / m \omega^{2}}=\frac{\Delta}{\pi\left(1-S / m \omega^{2}\right)}
$$

\section{Analysis of Forced and Free Oscillations of a Generalized Maxwell Body}

A generalized Maxwell body consisting of $m+1$ springs and dashpots as shown in Figure 1, has the same deformation $\gamma$ in each
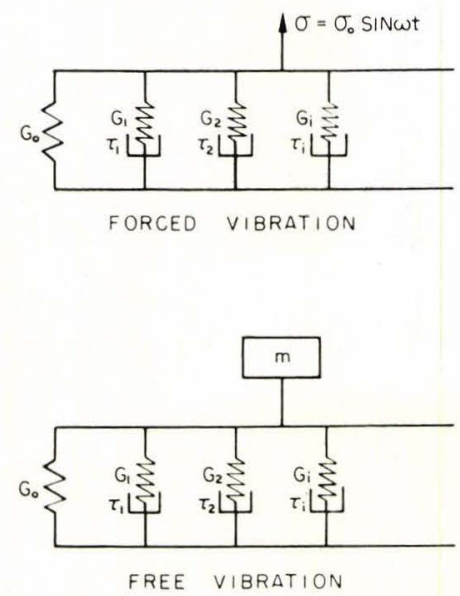

Fig. 1. Generalized Maxwell model. 
element while the stress $\sigma_{i}$ in the $i$ th element is related to the rate of deformation through the relation

$$
\dot{\gamma}=\frac{\dot{\sigma}_{i}}{G_{i}}+\frac{\sigma_{i}}{G_{i} \tau_{i}}
$$

where $G_{i}$ is the shear modulus (spring constant) and $\tau_{i}$ the relaxation time of the $i$ th member. The total stress on the model is

$$
\sigma=\sum_{i=0}^{n} \sigma_{i}
$$

Performing a Iaplace transformation on eqs. (2.1) and (2.2)

$$
p \bar{\gamma}-\gamma(0)=\frac{1}{G_{i}}\left(p+1 / \tau_{i}\right) \bar{\sigma}_{i}-\frac{\sigma_{i}(0)}{G_{i}}
$$

and

$$
\bar{\sigma}=\sum_{i=0}^{n} \bar{\sigma}_{i}
$$

is obtained.

In eq. (2.3) $\gamma(0)$ is the initial strain and $\sigma_{i}(0)$ the initial stress in the $i$ th element.

Substituting eq. (2.3) into eq. (2.4) and solving for $\bar{\sigma}$

$$
\bar{\sigma}=\bar{\gamma} \sum_{i=0}^{n} \frac{p G_{i}}{p+1 / \tau_{i}}+\sum_{i=0}^{n} \frac{\sigma_{i}(0)-G_{i} \gamma(0)}{p+1 / \tau_{i}}
$$

is obtained.

The complex shear modulus, which is the coefficient of $\bar{\gamma}$, may be obtained in its real and imaginary form by replacing $p$ with $j \omega$

$$
\begin{aligned}
G(p) & =\sum_{i=0}^{n} \frac{p G_{i}}{p+1 / \tau_{i}} \\
G^{*}(j \omega) & =\sum_{i=0}^{n} \frac{j \omega G_{i}}{j \omega+1 / \tau_{i}}
\end{aligned}
$$

and

$$
G_{r e}^{*}=\sum_{i=0}^{n} \frac{\left(\omega \tau_{i}\right)^{2} G_{i}}{1+\left(\omega \tau_{i}\right)^{2}}
$$




$$
G^{*}{ }_{i m}=\sum_{i=0}^{n} \frac{\omega \tau_{i} G_{i}}{1+\left(\omega \tau_{i}\right)^{2}}
$$

from which

$$
\left|G^{*}\right|=\left(G^{*}{ }_{r e}^{2}+G^{*}{ }_{i m}{ }^{2}\right)^{1 / 2}
$$

and

$$
\tan \delta=G^{*}{ }_{i m} / G^{*}{ }_{r e}
$$

Attaching a mass $m$ and an elastic spring $S$ to the model applying a forcing function $f(t)$ and assuming nonzero initial conditions $\gamma(0)$ $=\gamma_{0}$ and $\dot{\gamma}(0)=\dot{\gamma}_{0}$ the motion will be described by the differential squation

$$
m \ddot{\gamma}+\sigma+\gamma S=f(t)
$$

or after Laplace transformation

$$
m p^{2} \bar{\gamma}-m p \gamma_{0}-m \dot{\gamma}_{0}+\bar{\sigma}+\bar{\gamma} S=F(p)
$$

Substituting $\bar{\sigma}$ from eq. (2.5) and solving for $\bar{\gamma}$

$$
\gamma=\frac{F(p)+m p \gamma_{0}+m \dot{\gamma}_{0}+\sum_{i=0}^{n} \frac{G_{i} \gamma_{0}-\sigma_{i}(0)}{p+1 / \tau_{i}}}{S+m p^{2}+\sum_{i=0}^{n} \frac{p G_{i}}{p+1 / \tau_{i}}}
$$

results.

For free vibrations $F(p)=0$. Multiplying both the numerator and denominator of eq. (2.14) by $\prod_{i=0}\left(p+1 / \tau_{i}\right), \bar{\gamma}$ may be expressed as the ratio of two polynomials

$$
\bar{\gamma}=\frac{g(p)}{h(p)}
$$

where $h(p)$ is a polynomial of order $n+2$.

The inverse transform of eq. (2.15) yields the deformation of the model. Considering a system with less than critical damping, the motion will consist of a damped sinusoidal vibration superimposed on several creep terms. ${ }^{9}$

Because only one mass is attached to the model the system has a single degree of freedom and will vibrate at a single frequency $\omega$. 
Consequently, eq. (2.15) when expanded into $n+2$ partial fractions will produce two terms with complex conjugate and $n$ terms with real denominators

$$
\begin{aligned}
\bar{\gamma}=\frac{g(p)}{h(p)}=\frac{a_{0} \omega}{(p+k)^{2}+a_{1}(p+k)}+\frac{a_{2}}{p+\theta_{2}} \\
+\frac{a_{3}}{p+\theta_{3}}+\ldots+\frac{a_{n+2}}{p+\theta_{n+2}}
\end{aligned}
$$

and after inversion.

$$
\gamma=A e^{-k t} \sin (\omega t+\psi)+a_{2} e^{-\theta_{2} t}+\ldots+a_{n+2} e^{-\theta_{n+2} t}
$$

where $k$ and $\omega$ are the real and imaginary parts of the single pair of complex roots and $\theta_{2} \ldots \theta_{n+2}$ the $n$ real roots of the polynomial $h(p)$.

The values of $k$ and $\omega$ are independent of the initial conditions and, therefore, the complex modulus can be determined directly.

Varying the applied mass, $m$, and spring constant, $S$, the logarithmic decrement $\Delta=2 \pi k / \omega$ can be computed as a function of the frequency $\omega$ for any given set of $G_{i}$ and $\tau_{i}$. These values may then be substituted into eq. (1.9) to obtain $\lambda$ which in turn may be used to compute three different values of $G$ from eqs. (1.7), (1.10), and (1.12) and three values of the damping, $\tan \delta$, from eqs. (1.8), (1.11), and (1.13). These may then be compared with $G^{*}{ }_{r e}$ and $\tan \delta$ of eqs. (2.8) and (2.11) which are based on a forced vibration experiment.

\section{Numerical Comparison}

The method is illustrated for a standard solid consisting of a spring $\left(G_{0}=200 \mathrm{psi}\right)$ and a Maxwell element $\left(\tau_{1}=1 \mathrm{sec}, G_{1}=800 \mathrm{psi}\right)$ in parallel. Results are plotted as functions of the angular frequency $\omega$ in Figure 2.

It is to be noted that for small damping the free and forced moduli and $\tan \delta$ coincide. Appreciable differences occur for increasing damping and especially for values greater than 0.6.

The inclusion of the $\lambda^{2} / 4$ terms, with a minus sign in eqs. (1.7) and (1.8) creates large errors in both modulus and damping. ${ }^{8}$ When it is used with a plus sign in eqs. (1.10) and (1.11) the damping, $\tan \delta$, obtained from forced vibration computations [eq. (2.11)] is well approximated but the modulus, $G^{+}$, is considerably overestimated 

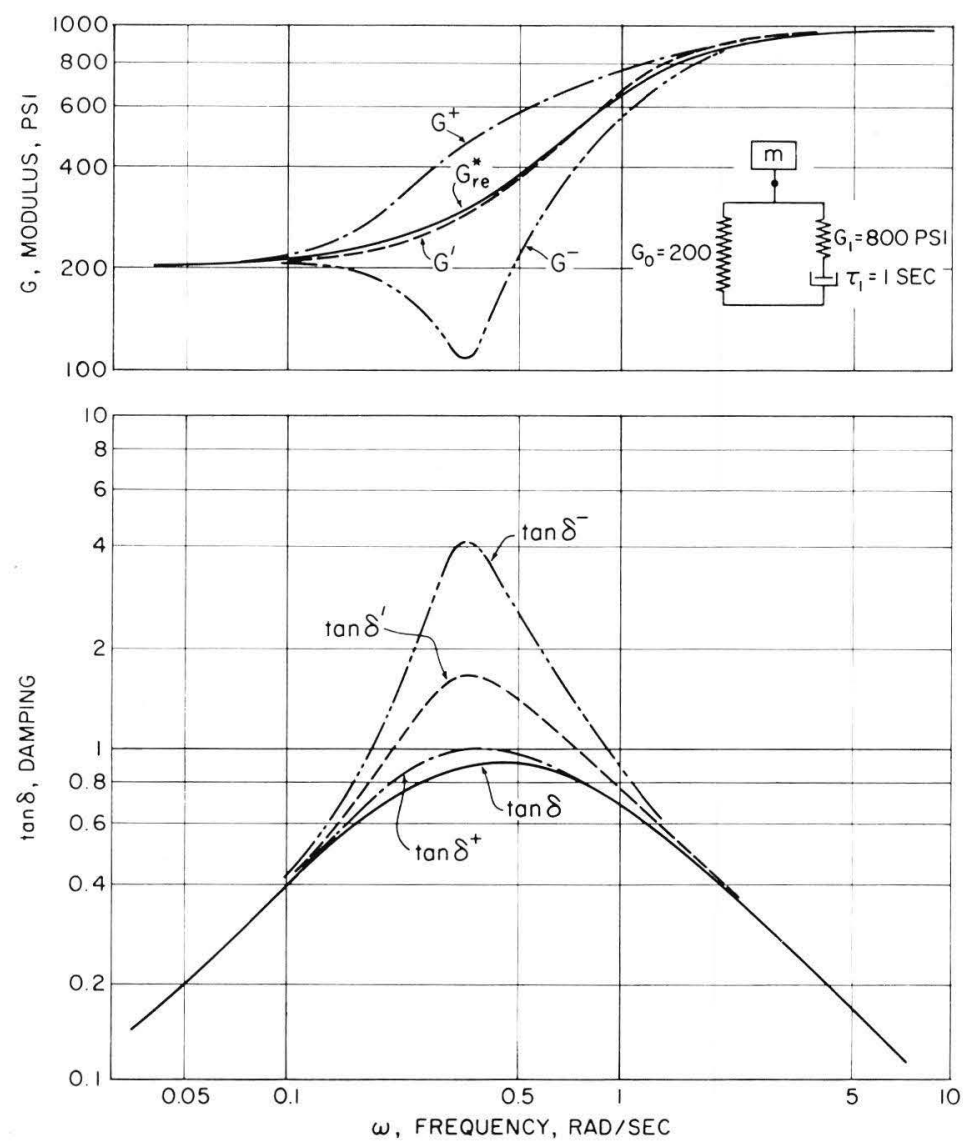

Fig. 2. Modulus and damping of standard solid.

ompared with $G^{*}{ }_{r e}$. The simplified quantities of eqs. (1.12) and (1.13) without the square term provide a better approximation for the modulus while the damping is too high.

As damping values greater than 0.3 are very difficult to measure with some precision, the use of an additional elastic element is a necessity. In Figure 3 results are shown for the model of Figure 2 with an additional spring $(S=1200 \mathrm{psi}$ ) with which the apparent $\tan \delta$ $=\Delta / \pi$ of the complete system stays below 0.26 . It is observed that the error is considerably smaller in the transition region. Using the 

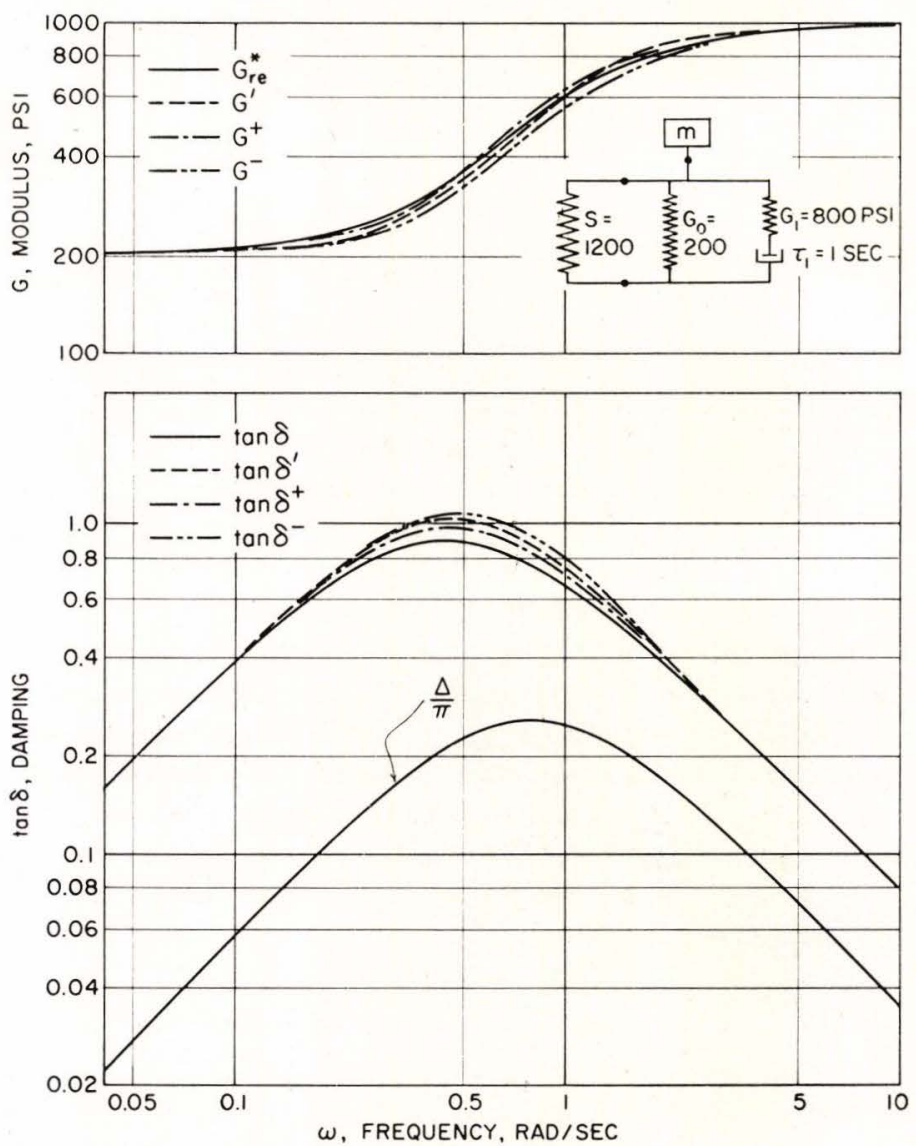

Fig. 3. Modulus and damping of the standard solid shown in Fig. 2 with added elastic member for free vibrations.

simplified eqs. (1.12) and (1.13) it is less than $16 \%$ for the damping and less than $6 \%$ for the real part of the modulus. Because the overall damping is small the inclusion of the square term has no appreciable effect.

The region in which an added stiffness can be used in practice is limited because a measurement uncertainty in $\Delta$ is amplified in the same proportion as the damping is reduced [see eq. (1.13)]. The practical limit of this method is around a material damping of 3 . 
TABLE I

Parameters Of Generalized Maxwell Model

\begin{tabular}{rrr}
\hline$i$ & $G_{i}$, psi & $\tau_{i}$, sec \\
\hline 0 & 200 & $\infty$ \\
1 & 200 & 10 \\
2 & 400 & 1.0 \\
3 & 600 & $10^{-1}$ \\
4 & 800 & $10^{-2}$ \\
5 & 2,200 & $10^{-3}$ \\
6 & 6,200 & $10^{-4}$ \\
7 & 25,600 & $10^{-5}$ \\
8 & 141,000 & $10^{-6}$ \\
\hline
\end{tabular}

The general trend of these results conforms to those of Markovitz and Parke $\mathrm{e}^{5,6}$ obtained on a standard solid.

A second example is given for a real material.

A large number of free damped vibration experiments were previously conducted on a potassium chloride filled polyurethane elastomer to determine its complex shear modulus and damping over a wide range of frequencies. ${ }^{10} \quad$ A generalized Maxwell model, consisting of a spring and eight spring-dashpot elements in parallel, was fitted to the test results using Tobolsky's method. ${ }^{11}$ The obtained $G$ and $\tau$ values are listed in Table $\mathrm{I}$.

These parameters were then introduced into eqs. (2.8) and (2.11) to determine the forced motion modulus and damping and into eqs. (1.7)-(1.13) for the free damped values.

The solutions of the $n+2=10$ th-order polynomial equation, $h(p)$, was carried out on an IBM 7094 computer for various values of $m$. The computations have in each case, as expected, produced a single set of complex conjugate roots and therefore only one value of frequency $\omega$ for each value of $m$.

The computed quantities are compared in Figure 4. The results indicate a similar trend as those of the three-element model.

\section{Conclusions}

An analysis of a generalized Maxwell model, representing a viscoelastic solid under conditions of forced and free oscillations shows that the complex moduli computed from the two types of motions are not identical. For low damping the approximation provided by a free- 

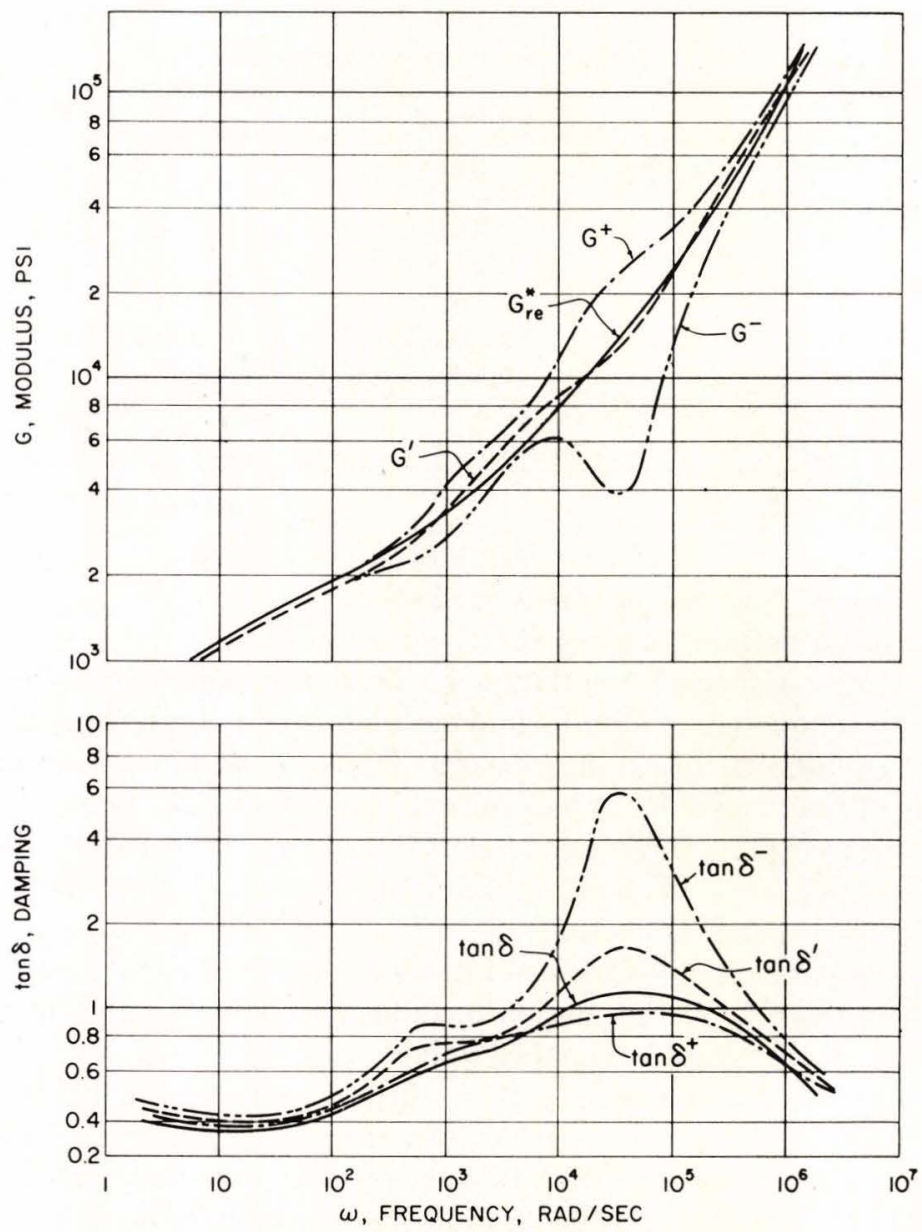

Fig. 4. Modulus and damping of a generalized Maxwell model shown in Fig. with constants listed in Table I.

vibration experiment is good. The deviations stay below $20 \%$ when the logarithmic decrement of the oscillatory system, which if needed is provided with an additional elastic element, is less than 0.6.

Damping values are improved by the inclusion of the square of the logarithmic decrement in eq. (1.11), while a better approximation of the modulus is obtained when this term is neglected. 
This research has been supported by the U. S. Office of Naval Research under contract No. NONR 266(78) and carried out at the Department of Civil Engineering, Columbia University.

\section{References}

1. D. R. Bland, The Theory of Linear Viscoelasticity, Pergamon, New York, 1960 .

2. F. R. Schwarzl and A. J. Staverman, J. Appl. Phys., 23, 838 (1952).

3. S. Neumark, Concept of Complex Stiffness Applied to Problems of Oscillations with Viscous and Hysteretic Damping, Royal Aircraft Establishment Report No. Aero. 2592, 1957.

4. A. J. Staverman and F. R. Schwarzl, "Linear Deformation Behavior of High Polymers," in Die Physik der Hochpolymeren, Vol. IV, H. A. Stuart, Ed., Springer-Verlag, Berlin, 1956.

5. H. Markovitz, J. Appl. Phys., 22, 21 (1963).

6. S. Parke, Brit. J. Appl. Phys., 17, 271 (1966).

7. R. Buchdahl, "The Rheology of Organic Glasses," in Rheology, F. Eirich, Ed., Academic Press, New York, 1958, p. 148.

8. N. W. Tschoegl, J. Appl. Phys., 32, 1792 (1961).

9. A. S. Elder, Trans. Soc. Rheol., 9:2, 187 (1965).

10. T. Nicholas and R. A. Heller, Exptl. Mech., 7:3, 110 (1967).

11. A. V. Tobolsky, Properties and Structure of Polymers, Wiley, New York, 1960. 